\title{
The effect of oral ondansetron on QT interval in children with acute gastroenteritis; a retrospective observational study
}

\author{
Heewon Yang ${ }^{1}$, Woochan Jeon ${ }^{2}$, Yura Ko ${ }^{1}$, Sooin Jeong ${ }^{3}$ and Jisook Lee ${ }^{1 *}$
}

\begin{abstract}
Background: In mildly to moderately dehydrated patients with acute gastroenteritis (AGE), oral rehydration therapy (ORT) is the treatment of choice. Though ondansetron is a very effective antiemetics and leads to succeed ORT, there have been reports QT prolongation in patients using it. We investigated the effect of oral ondansetron on QT interval in mildly to moderately dehydrated children with AGE.

Methods: This retrospective observational study was conducted in a single pediatric emergency department (ED) of a tertiary university hospital. We collected the medical records of patients with a primary diagnosis of AGE who received oral ondansetron and underwent an electrocardiogram between January 2017 and June 2018. A pediatric emergency physician calculated the corrected QT interval (QTc) by Bazett's method, and the calculations were reviewed by a pediatric cardiologist. QTc values before (preQTc) and after (postQTc) ondansetron administration were analyzed. $\triangle$ QTc was calculated as the change from preQTc to postQTc. We also investigated any cardiac complications from oral ondansetron.
\end{abstract}

Results: Total 80 patients were included. The mean age of the patients was $53.31 \pm 32.42$ months, and $45 \%$ were male. The mean dose of oral ondansetron was $0.18 \pm 0.04 \mathrm{mg} / \mathrm{kg}$. The mean interval from administration of ondansetron to performance of the electrocardiogram was $65 \pm 26 \mathrm{~min}$. The mean preQTc was $403.3 \pm 24.0 \mathrm{~ms}$, and the mean postQTc was $407.2 \pm 26.7 \mathrm{~ms}$. Two patients had a preQTc $\geq 460 \mathrm{~ms}$, and one patient had a postQTc $\geq 460 \mathrm{~ms} . \triangle \mathrm{QTc}$ was $\geq 30 \mathrm{~ms}$ in seven patients (8.8\%). No $\Delta \mathrm{QTc}$ was $\geq 60 \mathrm{~ms}$. No pre- or postQTc was $\geq 500 \mathrm{~ms}$. No patient had a fatal cardiac arrhythmia after taking ondansetron.

Conclusion: Oral administration of a single dose of ondansetron in children with AGE did not cause high-risk QTC prolongation or fatal arrhythmia.

Keywords: Ondansetron, QT prolongation, Vomiting, Child, Emergency department

\section{Background}

Acute gastroenteritis (AGE) is one of the most common diseases of children, with 1.3 million deaths worldwide annually and about 500,000 deaths of children under

\footnotetext{
*Correspondence: eesysook@naver.com

${ }^{1}$ Department of Emergency Medicine, Ajou University School

of Medicine, 164, World cup-ro, Yeongtong-gu, Suwon 16499, Republic

of Korea

Full list of author information is available at the end of the article
}

5 years old [1]. The World Health Organization, the US Centers for Disease Control and Prevention, and the American Academy of Pediatrics have recommended oral rehydration therapy (ORT) as initial treatment in mildly to moderately dehydrated children. Successful treatment with ORT reduces the need for intravenous (IV) fluid therapy and can reduce the duration of hospital stay and prevent unnecessary use of medical resources $[2$, 3]. Vomiting control is pivotal in the treatment of mildly 
to moderately dehydrated children with AGE. In the emergency department (ED), vomiting is a major obstacle to the use of ORT. Recent studies have recommended the use of oral ondansetron as an antiemetic [4].

Ondansetron is an antagonist of 5-hydroxytryptamine type 3 and may cause QT prolongation due to its mechanism of action. Many studies have reported that ondansetron can enable the successful use of ORT by suppressing vomiting [2, 5-8]. Ondansetron has also been safely and effectively used in patients with cancer and postoperative patients [9]. However, the US Food and Drug Administration reported in 2011 that IV ondansetron possibly caused fatal arrhythmias in patients with prolonged QT interval [10]. On the other hand, recent studies found that IV ondansetron did not cause QT prolongation or increase the risk of fatal arrhythmias in mildly to moderately dehydrated pediatric patients with AGE $[9,11,12]$.

For convenience, oral ondansetron has been preferred to IV ondansetron to treat children with vomiting while receiving ORT [13]. Most previous studies have focused on the safety of IV ondansetron, and there have been no studies of changes in QT interval after oral administration of ondansetron. Therefore, we conducted a study to investigate the effect of oral ondansetron on QT interval in mildly to moderately dehydrated children with AGE.

\section{Methods}

\section{Patients and setting}

This retrospective observational study was conducted in a pediatric ED of Ajou University Hospital which is the largest tertiary referral university hospital located on Southern Kyounggi Province. We reviewed the charts of all patients whose primary discharge diagnosis was AGE between January 2017 and June 2018. Among them, we selected patients aged from 6 months to 14 years. Finally, we included patients who received oral ondansetron with ORT and underwent an electrocardiogram (ECG) according to the ORT protocol in our pediatric ED. Patients were excluded if they had clinical dehydration scale $(\mathrm{CDS}) \geq 6$, oliguria, surgical abdomen, medical history of abdomen surgery, congenital heart disease, arrhythmias, or a history of use of QT-prolonging medication [14]. We also excluded patients with missing medical records, those who could not measure the QT intervals due to ECG noise and those who received intravenous fluid or other antiemetics. The study was approved by institute' ethics committee. The study also complied with the principles of the Helsinki Declaration.

\section{ORT protocol}

In our pediatric ED, the ORT protocol with oral ondansetron has been performed for mildly to moderately dehydrated patients with CDS $\leq 5$ with AGE. If the patient complains of vomiting, $2 \mathrm{mg}$ (for patients $8-15 \mathrm{~kg}$ ), $4 \mathrm{mg}$ (for patients $15-30 \mathrm{~kg}$ ), or $8 \mathrm{mg}$ (for patients $>30 \mathrm{~kg}$ ) of ondansetron (Zofran Zydis ${ }^{\circledR}$, Novartis, Basel, Switzerland) was administered by the oral route for antiemetic effect [2]. ORT was started 15 to 20 min after administration of oral ondansetron. For early detection of QT prolongation and fatal arrhythmias, ECG recording (lead II; standard paper speed of $25 \mathrm{~mm} / \mathrm{sec}$, standard calibration of $10 \mathrm{~mm} / \mathrm{mV}$ ) was performed using biphasic defibrillator (Philips Medical Systems, Andover, MA, USA) before the administration of ondansetron. To detect any changes in the ECG after administration of ondansetron, a postECG was obtained before discharge from ED.

In this study, a pediatric emergency physician received $6 \mathrm{~h}$ of training from a pediatric cardiologist in the interpretation of pediatric ECGs, in particular, of QT interval measurement. The pediatric emergency physician calculated the corrected QT interval (QTc) by Bazzet's method $[15,16]$. All calculated QTcs were reviewed by the pediatric cardiologist. The pediatric emergency physician and the cardiologist were blinded to whether pre or post-ECG to take ondansetron and analysis of all results. Following the recommendation of a pediatric cardiologist, QT measurement was made in lead II and QT interval was determined as a mean value derived from at least 3 to 5 heartbeats [16]. We also measured the QT interval using the tangent method originally described by Lepeschkin and Surawicz [17]. No prominent $U$ waves or inverted $U$ waves were found on the ECGs.

We summarized the demographic characteristics of the patients, the dose of ondansetron according to body weight, the interval before performing post-ECG, and the parameters of QTc intervals. Also, we reviewed any adverse effects staying the ED.

Our primary outcome was the change in QTc interval $(\Delta \mathrm{QTC})$ after ondansetron administration. The definition of QT prolongation was $460 \mathrm{~ms}$ and QTc $\geq 500 \mathrm{~ms}$ indicated marked QT prolongation [16, 18]. According to previous "thorough QT study" criteria, $\Delta$ QTc $\geq 30 \mathrm{~ms}$ is defined as a "possible" cause for concern and $\Delta \mathrm{QTc}$ $\geq 60 \mathrm{~ms}$ as a "definite" cause for concern $[12,19,20]$. The secondary outcome was adverse electrical cardiac events after drug administration.

\section{Data analysis}

Continuous variables following normal distribution were expressed as means \pm standard deviation. Variables that were not found to follow a normal distribution were expressed as medians and interquartile ranges. Categorical variables were expressed as frequencies and percentages. A comparison of QTc before and after ondansetron administration was analyzed by Student's paired $t$-test and Mann-Whitney $U$ test on the resulting sample 
values. SPSS version 15.0 (SPSS, Chicago, IL, USA) was used for all statistical analyses. A $p$ value $<0.05$ was considered to indicate a significant difference.

\section{Results}

A total of 80 children were included in the analysis (Table 1). The mean age of the children was $53.3 \pm 32.4$ months (range, 7 to 161 months), and $45 \%$ were male. The mean weight was $18.9 \pm 10.0 \mathrm{~kg}$. The mean number of vomiting was $2.5 \pm 2.2$. No patients complained bloody or bilious vomiting. Six patients reported one or two events of diarrhea. The average of CDS was 2 score. The mean dose of ondansetron was $0.18 \pm 0.04 \mathrm{mg} / \mathrm{kg}$. The mean interval from oral administration of ondansetron to performance of the ECG was $65 \pm 26 \mathrm{~min}$. The mean length of stay in the ED was $147 \pm 45 \mathrm{~min}$. According to ED protocol, all of 80 patients received only single dose of ondansetron with successful ORT. No patient was treated by IV hydration or got blood tests other than ORT. The median QTc at baseline (preQTc) and after taking ondansetron (postQTc) was $403.3 \pm 24.0$ and $407.2 \pm 26.7 \mathrm{~ms}$, respectively; the difference was not significant $(p=0.08)$ (Table 2$)$.

We analyzed the changes in QT interval before and after administration of oral ondansetron (Fig. 1). No

Table 1 Baseline characteristics (80 patients)

\begin{tabular}{lll}
\hline & Mean \pm SD & Range \\
\hline Male & $36(45 \%)$ & \\
Age, months & $53.3 \pm 32.4$ & $7-161$ \\
Weight, $\mathrm{kg}$ & $18.9 \pm 10.0$ & $8.0-67.9$ \\
Number of vomiting & $2.5 \pm 2.2$ & $1-10$ \\
Clinical dehydration scale & $2.0 \pm 1.1$ & $2-5$ \\
Ondansetron, mg/kg & $0.2 \pm 0.0$ & $0.1-0.2$ \\
Time to take post-ECG, min & $65 \pm 26$ & $30-192$ \\
Length of stay in ED, min & $147 \pm 45$ & $143-338$ \\
\hline Data are presented as number $(\mathrm{n}, \%)$ or mean \pm standard deviation (SD) with \\
range & & \\
ED emergency department & &
\end{tabular}

Table 2 The effect of oral ondansetron on QT interval

\begin{tabular}{llll}
\hline & Mean \pm SD & Range & \\
PreQTc, ms & $403.3 \pm 24.0$ & $343.0-482.0$ & \\
PostQTc, ms & $407.2 \pm 26.7$ & $343.0-472.0$ & \\
Paired t-test & & & $\mathbf{9 5 \% ~ C l}(\boldsymbol{P}$ value) \\
$\Delta \mathrm{QTc}$ ms & $3.9 \pm 19.9$ & $-40.0-47.0$ & $-0.5-8.3(0.08)$ \\
\hline
\end{tabular}

Data are presented as mean with standard deviation (SD), Range and Confidential interval (CI). QTc corrected QT interval

QTc is calculated by Bazett's method

$\triangle Q T c$ is the change of QT interval before and after oral ondansetron administration patient had a prolonged QTc $\geq 500 \mathrm{~ms}$. Only two patients had baseline QTc exceeding $460 \mathrm{~ms}$. All of them had no underlying disease and did not take QT prolonging medications. Patient 1 was a 17 -month-old girl. She weighed $11 \mathrm{~kg}$ and received $2 \mathrm{mg}$ of oral ondansetron. An ECG was tested within an hour and 8 minutes of the ondansetron administration. Her QTc increased from $467 \mathrm{~ms}$ to $472 \mathrm{~ms}$ with normal sinus rhythm. Length of stay in ED was $3 \mathrm{~h} 05 \mathrm{~min}$. Patient 2 was a 40 -month-old girl. She weighed $15.8 \mathrm{~kg}$ and received $4 \mathrm{mg}$ of oral ondansetron. After $58 \mathrm{~min}$ we obtained ECG of the patient 2 . QTc was decreased from $462 \mathrm{~ms}$ to $435 \mathrm{~ms}$ with a normal sinus rhythm. Length of stay in ED was $2 \mathrm{~h} 15 \mathrm{~min}$. After ORT, there was no abnormal rhythm or vital signs. They improved initial symptoms and were safely discharged. A comparison of QTc before and after ondansetron administration $(\Delta \mathrm{QTc})$ was performed (Fig. 2). The mean $\Delta \mathrm{QTc}$ was $3.9 \mathrm{~ms}$ (range, -40.0 to $47.0 \mathrm{~ms}$ ). Seventy-three children $(91.3 \%)$ had a $\Delta$ QTc $<30 \mathrm{~ms}$, and 7 children $(8.7 \%)$ had a $\Delta \mathrm{QTc} \geq 30 \mathrm{~ms}$. No child had a prolonged $\Delta \mathrm{QTc}$ $\geq 60 \mathrm{~ms}$. To analyze the relationship between $\Delta \mathrm{QTc}$ and the dose of oral ondansetron according to weight, we divided the patients into two groups according to whether $\triangle \mathrm{QTc}$ was greater or less than $30 \mathrm{~ms}$ (Table 3). Patients with $\Delta \mathrm{QTC} \geq 30 \mathrm{~ms}$ were younger than patients with $\Delta$ QTc $<30 \mathrm{~ms}$ (median [IQR], 30.0 [10.0 - 44.0] months vs 52.0 [31.5 - 68.3] months, $P=0.02$ ). Although post-ECG $\triangle \mathrm{QTC}$ did not differ between the two groups, $\triangle$ QTc of baseline ECG was shorter in the group with $\Delta \mathrm{QTc} \geq 30 \mathrm{~ms}$ (median [IQR], 383.0 [379.0 - 394.0] ms vs $404.0[387.8-424.0] \mathrm{ms}, p=0.04)$. Seven patients show QT prolongation more than $30 \mathrm{~ms}$. All of them were previous healthy children. Among them, 5 patients (71\%) were female. The median dose of ondansetron per weight (kilogram) and the interval between administration of ondansetron and performing the post-ECG did not significantly differ between the two groups. During the stay in the ED, there were no adverse cardiac events or fatal arrhythmias after taking the medicine.

\section{Discussion}

To the best of our knowledge, this study was the first to evaluate the effect of single dose oral ondansetron on changes in the QT interval. We showed that single dose oral ondansetron did not prolong the QT interval and resulted in effective use of ORT in children with AGE. A previous study reported that IV ondansetron did not affect the QTc of pediatric patients who were receiving ondansetron for nausea and vomiting [9]. According to the author's description, oral administration of ondansetron was crucial for ORT in mild to moderately dehydrated children. Though this study was retrospectively 


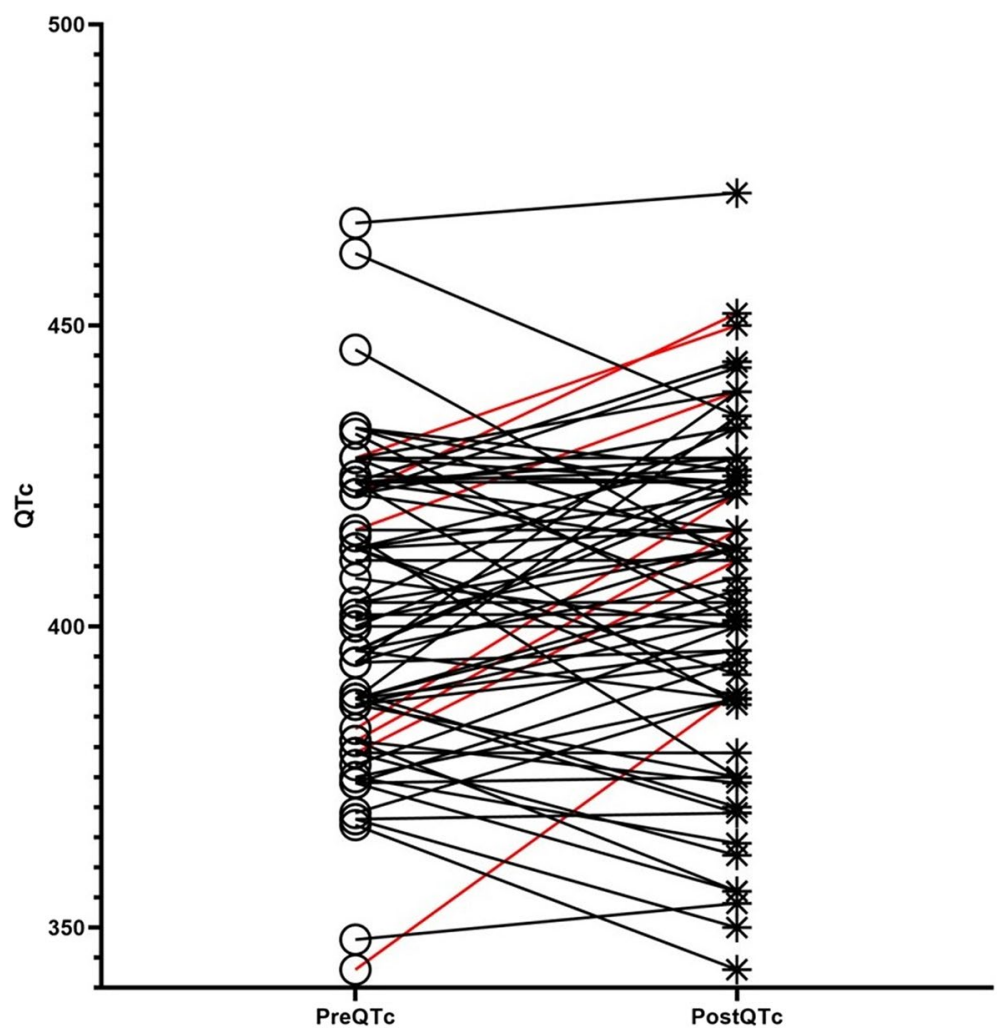

Fig. 1 Change of QT interval before and after oral ondansetron administration. Pre and Post QTc value before and after ondansetron administration, respectively; $\triangle \mathrm{QTC} \geq 30 \mathrm{~ms}$ (red); $\triangle \mathrm{QTc}$ is the change of $\mathrm{QT}$ interval before and after oral ondansetron administration

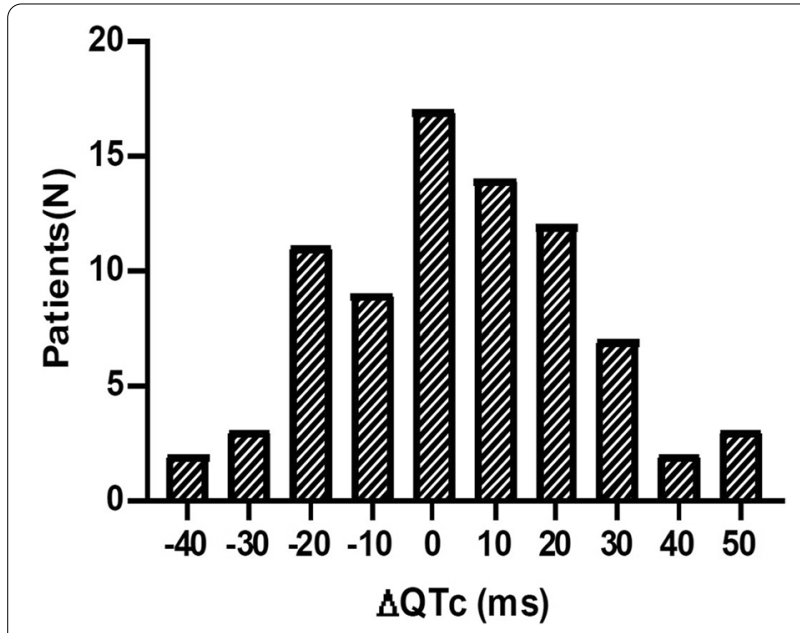

Fig. 2 The distribution of $\triangle \mathrm{QTC}$ by pre and post ondansetron effect

small sample sized, our results were noteworthy in this respect.

The patients in this study had a mean age of $53.3 \pm 32.4$ months, ranging from 7 to 161 months. This was an appropriate age group to receive ORT with oral
Table 3 Comparisons according to $\triangle \mathrm{QTC}$

\begin{tabular}{llll}
\hline & $\mathbf{\Delta Q T c}<\mathbf{3 0} \mathbf{~ m s}$ & $\mathbf{\Delta Q T c} \geq \mathbf{3 0 ~} \mathbf{m s}$ & $\boldsymbol{P}$ \\
\hline Patients, n (\%) & $73(91.3)$ & $7(8.7)$ & \\
Female, $\mathrm{n}(\%)$ & $39(53)$ & $5(71)$ & \\
Age, mon & $52.0(31.5-68.3)$ & $30.0(10.0-44.0)$ & 0.02 \\
Weight, kg & $17.0(13.0-20.0)$ & $12.0(10.6-15.6)$ & 0.02 \\
Ondansetron, mg/kg & $0.2(0.1-0.2)$ & $0.2(0.1-0.2)$ & 0.56 \\
PreQTc, ms & $404.0(387.8-424.0)$ & $383.0(379.0-394.0)$ & 0.04 \\
PostQTc, ms & $411.0(388.0-425.3)$ & $422.0(411.0-439.0)$ & 0.09 \\
\hline
\end{tabular}

Data are presented as number $(n, \%)$ or median with interquartile range (IQR) $\triangle \mathrm{QTC}$ is the change of QT interval before and after oral ondansetron administration

$P$ value $<0.05$ was set to be statistically significant

ondansetron. The size of the single dose of ondansetron varied according to the patient's weight: $2 \mathrm{mg}$ for patients $8-15 \mathrm{~kg}, 4 \mathrm{mg}$ for patients $15-30 \mathrm{~kg}$, and $8 \mathrm{mg}$ for patients $>30 \mathrm{~kg}$. The mean dose per unit weight was $0.18 \pm 0.04 \mathrm{mg}$. Because the bioavailability of the oral form of ondansetron is 56 to $60 \%$ of that of IV ondansetron due to first-pass metabolism, the size of the dose was adequate, considering the recommended IV dose 
of 0.1 to $0.15 \mathrm{mg} / \mathrm{kg}[9,12]$. The mean interval from oral administration of ondansetron to performance of the EGG was $65 \pm 26 \mathrm{~min}$. Since the time to reach peak blood concentration of ondansetron is 30 to $120 \mathrm{~min}$ after oral ingestion, our results showed the change in QTc at peak concentration of oral ondansetron [21].

A previous study of IV ondansetron in healthy children who were undergoing elective surgery under general anesthesia or who had AGE with mild dehydration found a nonsignificant prolongation of QTc of 0.4 to $17 \mathrm{~ms}$ [22]. Our results were similar, showing that oral ondansetron did not prolong QTc and that changes in $\Delta \mathrm{QTC}$ were not significant. However, Trivedi et al. reported that several risk factors, including electrolyte imbalance, multidrug use with the possible risk of QT interval change, and organ dysfunction, prolonged QT in pediatric patients in the intensive care unit [18]. In patients who had risk factors or baseline QTc over $460 \mathrm{~ms}$, QT could be prolonged more than $500 \mathrm{~ms}$. The patients we included were children with acute diseases who were previously healthy and without underlying disease and who received only a single dose of ondansetron. Recently two pediatric emergency centers in Pakistan conducted randomized, double-blind, placebo-controlled trial that supported our results which reported safety use and improve success rate of ORT of single dose oral ondansetron among children with gastroenteritis-associate vomiting [23]. Therefore, further multicenter prospective study focusing on the effect of oral ondansetron on QT prolongation is needed to deduce the efficacy and safety of repeated doses of ondansetron in patients with a high risk of QT prolongation.

The International Conference on Harmonization (ICH) findings have guided the US Food and Drug Administration on issues of drug-induced QT prolongation. The $\mathrm{ICH}$ has suggested that an increase in QTc of $30 \mathrm{~ms}$ is a "possible" cause for concern and an increase of $60 \mathrm{~ms}$ is a "definite" cause for concern. After administration of an at-risk drug, increases in QT or QTc to $\geq 500 \mathrm{~ms}$ or to $\geq 60 \mathrm{~ms}$ over baseline are commonly used as thresholds for potential discontinuation or alternative pharmacotherapy $[16,18]$. In our study, there were seven patients with an increase in QTc of $\geq 30 \mathrm{~ms}$ and no patients with an increase of $\geq 60 \mathrm{~ms}$. Patients with an increase of $\geq 30 \mathrm{~ms}$ were younger and had a lower body weight. In patients with an increase of $\geq 30 \mathrm{~ms}$, baseline QTc was shorter and the change of QTc after taking medicine was greater. The dose of ondansetron per unit weight and the interval from oral administration of ondansetron to performance of the EGG did not significantly differ between patients who had an increase of $<30 \mathrm{~ms}$ and those who had an increase of $\geq 30 \mathrm{~ms}$.. However, ondansetron might cause a greater prolongation of QTc in younger children.
Although all patients had QTc $<460 \mathrm{~ms}$ and there was no "definite" concern about $\Delta \mathrm{QTC}$, physicians need to be careful when using ondansetron in younger children.

Our study had several limitations. First, because it was a retrospective analysis, we could not analyze changes in QTc over time after oral ingestion of ondansetron. A previous study of IV ondansetron showed that the temporarily prolonged QTc became shorter over time after administration [9, 10]. Because the blood concentration of oral ondansetron peaks at 30 to $120 \mathrm{~min}$, further studies should measure QTc over this time range after oral administration. Second, the study had a small sample size. We retrospectively enrolled all patients who were satisfied with the inclusion criteria during the study period. We calculated statistical power analysis to determine sample size. According to the effect of intravenous ondansetron on QTc study among the children with AGE, incidence of $\Delta \mathrm{QTc}>30 \mathrm{~ms}$ was $7.5 \%$ after IV ondansetron administration [10]. Minimum number for adequate study power sample size was $8(80 \%$ power, $\alpha=0.05)$. We finally collected and analyzed 80 patient's data. Although this was retrospective study, our results with 80 participants could have power. However, being conducted in a single ED on acutely ill patients who were previously healthy, the results may not apply to the entire pediatric populations. In this pilot study, only one emergency physician measured QTc and one cardiology specialist confirmed. To improve data quality, we believe that two more physicians need to perform these measurements and check variability and reliability between observers in the further study. Third, there were no follow-up investigations such as ED revisit or outpatient visit. As a result, we were unaware of the long-term side effects. However, given the blood peak time of an oral ondansetron, we thought that the length of stay of ED was sufficient to observe side effects after oral administration. These limitations indicate that a larger prospective study is needed to explore the possibility of QT prolongation by oral ondansetron.

\section{Conclusion}

The effect of oral ondansetron on QT interval is one of the crucial subjects for its safety use among previously healthy children. Our study found that oral administration of a single dose of ondansetron did not result in a significant prolongation of QTc or fatal arrhythmias in mild to moderation dehydrated children with vomiting.

\section{Abbreviations}

AGE: Acute gastroengeritis; ORT: Oral rehydration therapy; CDS: Clinical dehydration scale; IV: Intravenous; ED: Emergency department; QTc: Corrected QT interval; IQR: Interquatile range. 


\section{Acknowledgements \\ Not applicable.}

\section{Authors' contributions}

HY and JL contributed to the study design, data collection. HY, WJ and SJ contributed to data review and analysis of results. $\mathrm{HY}$ and $J \mathrm{~L}$ contributed to writing of manuscript. WJ and YK contributed to review of manuscript drafts. All authors critically revised and edited the manuscript. The authors read and approved the final manuscript.

\section{Funding}

The authors have no financial support of the investigation or manuscript development.

\section{Availability of data and materials}

The study data is available from the corresponding author on reasonable request.

\section{Declarations}

\section{Ethics approval and consent to participate}

This study was conducted according to the guidelines laid down in the Declaration of Helsinki, and all procedures involving research study participants were approved by the Ajou Medical Center Institutional Review Board (approval number AJIRB-MED-MDB-20-123). Because of the study's retrospective nature, Ajou Medical Center Institutional Review Board specifically waived the requirement to obtain parental or guardian informed consent to conduct this study.

\section{Consent for publication}

Not Applicable.

\section{Competing interests}

All authors declare that they have no competing interests.

\section{Author details}

${ }^{1}$ Department of Emergency Medicine, Ajou University School of Medicine, 164, World cup-ro, Yeongtong-gu, Suwon 16499, Republic of Korea. ${ }^{2}$ Department of Emergency Medicine, Inje University, Ilsan Paik Hospital, Goyang, Republic of Korea. ${ }^{3}$ Division of Pediatric Cardiology, Department of Pediatrics, Ajou University School of Medicine, Suwon, Republic of Korea.

Received: 4 June 2021 Accepted: 11 October 2021

Published online: 10 November 2021

\section{References}

1. GBD 2015 Mortality and Causes of Death Collaborators. Global, regional, and national life expectancy, all-cause mortality, and causespecific mortality for 249 causes of death, 1980-2015: a systematic analysis for the Global Burden of Disease Study 2015. Lancet. 2016:388:1459-544.

2. Freedman SB, Adler M, Seshadri R, Powell EC. Oral ondansetron for gastroenteritis in a pediatric emergency department. N Engl J Med. 2006;354:1698-705.

3. Freedman SB, Steiner MJ, Chan KJ. Oral ondansetron administration in emergency departments to children with gastroenteritis: an economic analysis. PLoS Med. 2010;7(10):e1000350.

4. Rutman L, Klein EJ, Brown JC. Clinical pathway produces sustained improvement in acute gastroenteritis care. Pediatrics. 2017:140(4):e20164310.
5. Danewa AS, Shah D, Batra P, Bhattacharya SK, Gupta P. Oral Ondansetron in Management of Dehydrating Diarrhea with vomiting in children aged 3 months to 5 years: a randomized controlled trial. J Pediatr. 2016;169:105-9 e3.

6. Freedman SB, Powell EC, Nava-Ocampo AA, Finkelstein Y. Ondansetron dosing in pediatric gastroenteritis: a prospective cohort, dose-response study. Paediatr Drugs. 2010;12:405-10.

7. Marchetti F, Bonati M, Maestro A, Zanon D, Rovere F, Arrighini A, et al. Oral Ondansetron versus Domperidone for acute gastroenteritis in pediatric emergency departments: multicenter double blind randomized controlled trial. PLoS One. 2016;11:e0165441.

8. Ramsook C, Sahagun-Carreon I, Kozinetz CA, Moro-Sutherland D. A randomized clinical trial comparing oral ondansetron with placebo in children with vomiting from acute gastroenteritis. Ann Emerg Med. 2002;39:397-403.

9. Krammes SK, Jacobs T, Clark JM, Lutes RE. Effect of intravenous Ondansetron on the QT interval of Patients' electrocardiograms. Pediatr Emerg Care. 2018;34:38-41.

10. Hoffman RJ, Alansari K. Effect of intravenous ondansetron on QTC interval in children with gastroenteritis. Am J Emerg Med. 2018:36:754-7.

11. Freedman SB, Uleryk E, Rumantir M, Finkelstein Y. Ondansetron and the risk of cardiac arrhythmias: a systematic review and postmarketing analysis. Ann Emerg Med. 2014;64:19-25.e6.

12. Zuo P, Haberer LJ, Fang L, Hunt TL, Ridgway D, Russo MW. Integration of modeling and simulation to support changes to ondansetron dosing following a randomized, double-blind, placebo-, and active-controlled thorough QT study. J Clin Pharmacol. 2014;54:1221-9.

13. Hervas D, Armero C, Carrion T, Utrera JF, Hervas JA. Clinical and economic impact of oral ondansetron for vomiting in a pediatric emergency department. Pediatr Emerg Care. 2012;28:1166-8.

14. Friedman JN, Goldman RD, Srivastava R, Parkin PC. Development of a clinical dehydration scale for use in children between 1 and 36 months of age. J Pediatr. 2004;145(2):201-7.

15. Food, Drug Administration USDoH, Human S. Guidance for Industry. E14 clinical evaluation of QT/QTc interval prolongation and proarrhythmic potential for non-antiarrhythmic drugs. https://www.fda.gov/media/ 71372/download. Updated October 2005. Accessed 20 Oct 2019.

16. Goldenberg I, Moss AJ, Zareba W. QT interval: how to measure it and what is "normal". J Cardiovasc Electrophysiol. 2006;17:333-6.

17. Lepeschkin E, Surawicz B. The measurement of the Q-T interval of the electrocardiogram. Circulation. 1952;6:378-88.

18. Trivedi S, Schiltz B, Kanipakam R, Bos JM, Ackerman MJ, Ouellette Y. Effect of Ondansetron on QT interval in patients cared for in the PICU. Pediatr Crit Care Med. 2016;17:e317-23.

19. Patanwala $A E$, Amini R, Hays DP, Rosen P. Antiemetic therapy for nausea and vomiting in the emergency department. J Emerg Med. 2010;39:330-6.

20. Moffett PM, Cartwright L, Grossart EA, O'Keefe D, Kang CS. Intravenous Ondansetron and the QT interval in adult emergency department patients: an observational study. Acad Emerg Med. 2016;23:102-5

21. Roila F, Del Favero A. Ondansetron clinical pharmacokinetics. Clin Pharmacokinet. 1995;29:95-109.

22. Mehta D, Sanatani S, Whyte SD. The effects of droperidol and ondansetron on dispersion of myocardial repolarization in children. Paediatr Anaesth. 2010;20:905-12.

23. Freedman SB, Soofi SB, Willan AR, Williamson-Urquhart S, Siddiqui E, Xie J, et al. Oral Ondansetron Administration to Dehydrated Children in Pakistan: A Randomized Clinical Trial. Pediatrics. 2019;144(6):e20192161.

\section{Publisher's Note}

Springer Nature remains neutral with regard to jurisdictional claims in published maps and institutional affiliations. 